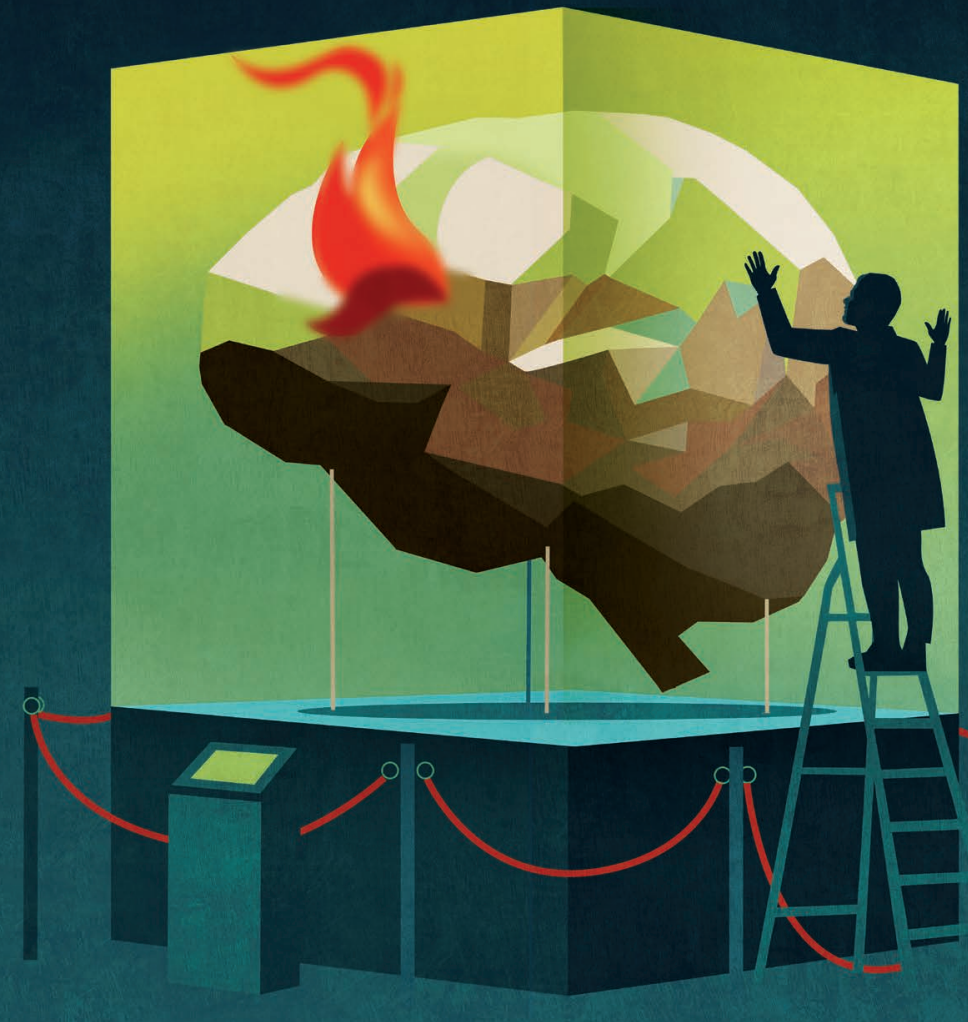

NEUROBIOLOGY

\title{
Across the divide
}

\author{
The blood-brain barrier represents a formidable \\ obstacle for cancer drugs.
}

\section{BY ERIC BENDER}

$\mathrm{T}$ The human brain has a huge appetite for glucose, and gobbles up about onefifth of the body's daily requirement. It is also hungry for oxygen and other nutrients, and responds rapidly to drugs such as alcohol and morphine. It even allows metastatic cancer cells to slip in from the bloodstream, using back doors that researchers do not yet understand.

But the brain is exceptionally good at keeping out most cancer drugs.

The blood-brain barrier (BBB), a unique assembly of blood vessels that filters what goes into and out of the brain, is responsible for this quirk. And it is also the main reason why treatments for cancer that work elsewhere in the body fail routinely when directed at the brain.

In fact, many studies of such drugs in people might be doomed from the start. "Clinical trials are run all the time with drugs that are known to not have good brain penetration," says Jann Sarkaria, a neuro-oncologist and radiologist at Mayo Clinic in Rochester,
Minnesota. "If we don't look at that brainpenetration issue, they're destined to fail."

The current treatment toolkit for brain tumours - surgery, radiation and chemotherapy - falls woefully short for most people with the condition, for whom survival time is often measured in months. Researchers and drug companies are struggling to find treatments that can cross the BBB to attack tumours while sparing the rest of the brain from injury.

"Everybody who is working in the braintumour field needs to get the therapeutic entities we're trying to develop into the brain," says Johanna Joyce, a cancer biologist at the University of Lausanne in Switzerland. "It's a topic that cuts across all of brain-tumour research."

\section{MEETING THE GATEKEEPER}

The brain is particularly sensitive to changes in its environment, which makes the BBB crucial for maintaining brain health. The endothelial cells that make up the walls of the BBB's vasculature are entwined so closely that most water-soluble and many lipid-soluble molecules are unable to pass through the space between them. They are wrapped in an unusually large number of cells called pericytes, which provide support to blood vessels. And such cells also interact with various other cells in the brain, including astrocytes, neurons and immune cells.

Describing the $\mathrm{BBB}$ as a barrier is somewhat misleading because the BBB is not a static wall. Instead, it actively pumps selected molecules into or out of the brain. Various types of transporter protein take up essential substances such as glucose and iron from the blood, which they then carry through the pumps into the brain, says Elga de Vries, a neuroimmunologist at the VU University Medical Center in Amsterdam.

Even when drugs do manage to cross the $\mathrm{BBB}$, researchers need to work out how to keep them there. Many cancer drugs that make it into the brain are promptly kicked back out by active 'efflux' pumps, says William Elmquist, a pharmacokineticist at the University of Minnesota in Minneapolis, who is developing ways of tailoring specific drugs to overcome this problem. Molecularly targeted agents known as kinase inhibitors might be particularly affected, and a number of companies are working to address the efflux issue in brain-cancer drug candidates, Elmquist says.

As tumours begin to grow, however, cracks begin to form in the exquisitely organized structure of the BBB. Cancer cells secrete molecules that open up the tight junctions between endothelial cells and disrupt the pumps. At the same time, "The cancer cell starts taking over the blood vessel - sucking oxygen and nutrients the best it can, and just growing like crazy," says Paul Lockman, a pharmaceutical scientist at the West Virginia University School of Pharmacy in Morgantown who is investigating the effectiveness of various brain-cancer drug candidates. "The blood-brain barrier is fundamentally broken down in almost every single tumour in the central nervous system," he says.

A complete collapse of the BBB would enable drugs to slip into the brain with ease. Unfortunately, the regions of $\mathrm{BBB}$ that surround most tumours are still intact enough to prevent drugs from passing through in sufficient concentrations to wipe out the cancer. "Even in the presence of tumours, the barrier is really good at keeping things out," Lockman says.

That point has long been a source of controversy among researchers and surgeons. Magnetic resonance imaging (MRI) scans often show contrast agents - used to enhance the visibility of tissues of interest - flooding into brain tumours, which suggests that cancer drugs do the same. However, various analyses, as well as experience gained from numerous clinical trials, have shown that such images often overestimate the BBB's permeability. As Sarkaria and his colleagues explain, even if the BBB is disrupted, there will be regions of the tumour in which the BBB remains intact (J. N. Sarkaria et al. Neuro Oncol. 20, 184-191; 2018). Only by reaching 
into every nook of a tumour will drugs have a chance of curing aggressive brain cancers such as glioblastoma multiforme (GBM). Finding ways to smuggle cancer drugs across the $\mathrm{BBB}$ is therefore essential.

\section{CROSSING THE BORDER}

Researchers are now working on fresh strategies for slipping drugs through the BBB. Molecules that can slip across easily tend to be small and to have high solubility in lipids, says Lockman. That makes traversing the BBB a particular challenge for the large, lipid-insoluble biological drugs that have improved outcomes for many other types of cancer. For instance, the monoclonal antibody trastuzumab, which is used successfully to target breast cancers with mutations that lead to overexpression of the protein HER2, crosses the $\mathrm{BBB}$ at a vastly slower rate than do most neuroactive drugs - about 800,000 times slower than nicotine, for example, Lockman says.

One response is to package such drugs with a protein fragment that has been optimized to drive the pumps that transport molecules across the BBB. The agent $\mathrm{xB}^{3}-001$, developed by Bioasis Technologies in Richmond, Canada, consists of a fragment of an iron-transporting protein that is attached to trastuzumab, which helps the drug to cross the BBB to attack HER2-expressing breast cancer that has spread to the brain. The company is now seeking approval for a clinical trial.

Another tactic that could work for cargoes that comprise small-molecule or biological drugs is to develop carriers that can deliver them to their targets both efficiently and in good condition. Unlike small-molecule drug candidates, the performance of which must be assessed almost from scratch, designs that combine an approved cancer drug with various carriers can be steadily tested and tweaked, says chemical engineer Mark Davis at the University of Southern California in Los Angeles. His team, which has painstakingly evolved nanoparticle-containing agents that show clinical promise for the treatment of other tumour types, is now creating agents that combine proteins that help molecules to cross the BBB with anti-cancer drugs. Earlier generations of these carriers were good at crossing the BBB but less so at delivering their cargoes. In preclinical work aimed at tackling breast cancer that has spread to the brain, Davis says that his team is optimizing carriers that not only get through the $\mathrm{BBB}$, but then disintegrate immediately to release a combination of trastuzumab and a chemotherapeutic agent.

Researchers have also tried to physically pump drugs across the BBB, using a method called convection-enhanced delivery. When a drug is simply injected into the brain, it

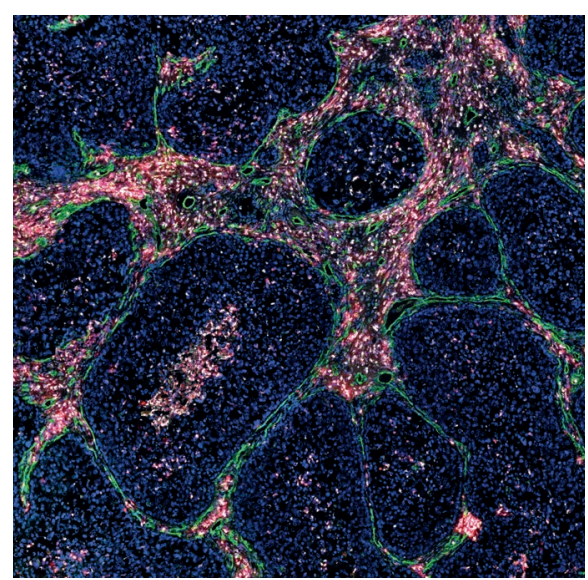

Blood vessels (green) in a secondary brain tumour.

hits the capillaries and is stopped almost immediately, Elmquist says. To help overcome this, researchers can insert multiport catheters that enable the drug to be infused into the brain under enough pressure to encourage it to flow into a tumour over many hours. Although phase III clinical trials failed to show efficacy in treating brain tumours, the strategy is being tested further using other drugs in at least ten early-phase trials.

\section{BEAMING IN}

Researchers in the field of brain cancer are keeping a close eye on clinical trials of focused ultrasound, a technique that builds on ultrasound-based diagnostic methods that are in widespread use and that offers the potential for a highly localized and non-invasive weakening of the BBB, enabling drugs to pass through.

Recipients are injected with microbubbles of gas that can open the BBB temporarily when hit by tightly controlled, low-power beams of ultrasound. This breach facilitates the passage of cancer drugs that are infused at the same time, says Kullervo Hynynen, a medical biophysicist at the University of Toronto in Canada.

The ultrasound beams create waves of pressure around the BBB. "When the highpressure phase comes, the bubble compresses much more than the surrounding fluid, because fluid is almost incompressible," Hynynen explains. "And then when the lowpressure phase comes, the bubble expands back. The blood-vessel walls expand and contract, and the tight junctions between the blood-vessel cells open. That is how we can increase the permeability."

Hynynen and his colleagues have already completed two small trials of the technique at Sunnybrook Health Sciences Centre in Toronto: one in people with cancer and the other in people with Alzheimer's disease. The method is safe and also seems to boost BBB permeability. The researchers plan to launch two further trials by the end of 2018. One study will use ultrasound to help deliver trastuzumab in people with breast cancer that has spread to the brain, and the other will combine the technique with chemotherapy drugs in those with GBM.
Part of the Sunnybrook procedure involves intensive scanning of the brain using MRI and computerized tomography to create detailed maps of tumours. On the basis of these maps, clinicians customize a helmet so that ultrasound transducers can be placed optimally for each patient. During treatment, real-time MRI scans are aligned with the maps. Microbubbles and the drug are then infused into the bloodstream, and focused ultrasound is used to boot the drug across the BBB.

Another early-stage trial, led by Alexandre Carpentier, a neurosurgeon at the Pitié Salpêtrière Hospital in Paris, is following a route that removes the need for patients to wear awkward headgear during treatment. Instead of using a helmet to generate ultrasound beams, Carpentier's group is seeking to combat GBM by implanting a small ultrasound transducer inside the skull after surgical removal of the tumour. The transducer is then used to activate microbubbles each time the patient is given chemotherapy, and the researchers have already reported encouraging results (A. Carpentier et al. Sci. Transl Med. 8, 343re2; 2016). Carpentier is a founder of Paris-based CarThera, a medical-device company that is developing such an implantable transducer.

Elisa Konofagou, a biomedical engineer at Columbia University in New York City, is leading the development of a procedure that does not even require real-time MRI scanning during the ultrasound treatment, and therefore avoids the need for combinations of expensive equipment that are available only in large medical centres.

By combining MRI and detailed modelling of the brain before treatment with passive monitoring by ultrasound during treatment, "we can know exactly whether we have the ultrasonic beam in the right region, overlapping with the bubbles", says Konofagou. Her group hopes to complete its first trial of the technique, in people with Alzheimer's disease, in 2019.

With the exception of the Pitié Salpêtrière system, these focused ultrasound techniques use beams that differ in frequency from those of standard clinical diagnostic systems, in part, because the beams must penetrate the skull. But similar to the clinical systems, all are lowenergy. "We are very gentle," Konofagou says. That is necessary to avoid breaking blood vessels, a risk that increases when such vessels are weakened by age or compromised by tumours, and the biggest safety concern associated with the technique.

"Focused ultrasound is a platform technology: a tool that people can use for many different therapies," says Hynynen. "We're trying to solve this problem once, so we won't have to solve it for everything. And we continue trying to make these treatment devices cheaper and easier to use."

Eric Bender is a freelance science writer in Newton, Massachusetts. 